MATHEMATICS OF GRAVITATION

PART I, LORENTZIAN GEOMETRY AND EINSTEIN EQUATIONS

BANACH CENTER PUBLICATIONS, VOLUME 41

INSTITUTE OF MATHEMATICS

POLISH ACADEMY OF SCIENCES

WARSZAWA 1997

\title{
INTEGRABILITY AND EINSTEIN'S EQUATIONS
}

\author{
N. M. J. WOODHOUSE \\ Wadham College, Oxford OX1 3PN, U.K. \\ E-mail: nwoodh@maths.ox.ac.uk
}

1. Introduction. In recent years, there has been considerable interest in Oxford and elsewhere in the connections between Einstein's equations, the (anti-) self-dual YangMills (SDYM) equations, and the theory of integrable systems. The common theme running through this work is that, to a greater or lesser extent, all three areas involve questions that can be addressed by twistor methods. In this paper, I shall review progress, with particular emphasis on the known and potential applications in relativity. Some of the results are well-established, others are more recent, and a few appear here for the first time.

2. Integrability and the SDYM equation. The first connection, between integrability and self-duality, comes from recognizing familiar integrable equations amongst the symmetry reductions of the SDYM equations (Ward 1985, 1990a). In null coordinates on complex Minkowski space, the metric is

$$
\mathrm{d} s^{2}=\mathrm{d} z \mathrm{~d} \tilde{z}-\mathrm{d} w \mathrm{~d} \tilde{w}
$$

and the equations are the curvature conditions

$$
F_{w z}=0, \quad F_{\tilde{z} \tilde{w}}=0, \quad F_{w \tilde{w}}=F_{z \tilde{z}}
$$

on a connection $\mathrm{D}=\mathrm{d}+\Phi$ on a vector bundle $E$ over an open subset $U$ of space-time. They can also be written in the 'Lax pair' form $[L, M]=0$, identically in $\zeta$, where

$$
L=\mathrm{D}_{w}-\zeta \mathrm{D}_{\tilde{z}}, \quad M=\mathrm{D}_{z}-\zeta \mathrm{D}_{\tilde{w}} .
$$

Under gauge transformations, that is, under changes in the local trivialization of the

1991 Mathematics Subject Classification: 83C60, 32Lxx.

The paper is in final form and no version of it will be published elsewhere. 
vector bundle, $L$ and $M$ go to $g L g^{-1}$ and $g M g^{-1}$, where $g$ is a function with values in the structure group, regarded here as a multiplication operator.

We make symmetry reductions by requiring that $\mathrm{D}$ should be invariant under a Lie algebra $\mathfrak{h}$ of conformal Killing vectors. That is, for each $X \in \mathfrak{h}$, there should be a Lie derivative operator $\mathcal{L}_{X}$ such that $\left[\mathcal{L}_{X}, \mathrm{D}\right]=0$. We can then write the equations as a reduced system on the quotient space of $U$ by the flow of $\mathfrak{h}$. The entire (proper) conformal group of complex space-time is the projective group $\mathrm{PGL}(4, \mathbb{C})$, which acts by mapping $X$ to a scalar multiple $A X A^{t}$, where $A \in \mathrm{GL}(4, \mathbb{C})$ is a representative of an element of the projective group and $X$ is a skew-symmetric $4 \times 4$ matrix with vanishing determinant. Here we identify the scalar multiples of $X$ with a point of space-time by writing

$$
X \propto\left(\begin{array}{cccc}
0 & s^{2} & -w & \tilde{z} \\
-s^{2} & 0 & -z & \tilde{w} \\
w & z & 0 & 1 \\
-\tilde{z} & -\tilde{w} & -1 & 0
\end{array}\right)
$$

where $s^{2}=z \tilde{z}-w \tilde{w}$. Thus a choice of a subgroup of GL(4, $\left.\mathbb{C}\right)$ gives a subgroup of the conformal group and thence a reduced system of equations.

There are many ways to make this explicit, depending on precisely which form of the self-duality equation one starts with. One more or less systematic method, at least for the reductions to one and two dimensions, is to use as dependent variables the Higgs fields $\phi_{X}=\mathrm{D}_{X}-\mathcal{L}_{X}$, which are sections of the adjoint bundle, represented by matrix-valued functions on space-time. In an invariant gauge, that is, one in which the Lie derivatives of $\Phi$ along the $X$ s vanish, the Higgs fields are simply the contractions of $\Phi$ with the generators of $\mathfrak{h}$. In most of the reductions to one and two dimensions, it is possible to choose a section of the orbits of $\mathfrak{h}$ on which the curvature vanishes. By choosing the gauge so that $\Phi$ vanishes on this section, one obtains $\mathrm{D}$ and the reduced equations in terms of the Higgs fields alone. The two following examples illustrate this, where we take the subgroups of $\mathrm{GL}(4, \mathbb{C})$ to be, respectively, the groups of matrices of the form

$$
\left(\begin{array}{cccc}
c & 0 & b & a \\
0 & c & -a & 0 \\
0 & 0 & c & 0 \\
0 & 0 & 0 & c
\end{array}\right), \quad\left(\begin{array}{cccc}
d & c & b & a \\
0 & d & c & b \\
0 & 0 & d & c \\
0 & 0 & 0 & d
\end{array}\right) .
$$

(Since the multiples of the diagonal act trivially in the projective group, these generate, respectively, two and three-dimensional abelian groups of conformal motions.)

Example 1 (Mason and Sparling 1989). The conformal Killing vectors are $X=$ $\partial_{w}-\partial_{\tilde{w}}$ and $Y=\partial_{\tilde{z}}$ (both are in fact translations). We can choose the gauge so that $\Phi=Q \mathrm{~d} \tilde{z}-P \mathrm{~d} \tilde{w}$, where the Higgs fields $P$ and $Q$ are functions of $x=w+\tilde{w}$ and $t=z$. Then the reduced equations are

$$
Q_{x}=[P, Q] \quad Q_{t}+P_{x}=0 .
$$


When the gauge group is $\mathrm{SL}(2, \mathbb{C})$, these are equivalent to the Korteweg de Vries $(\mathrm{KdV})$ or the nonlinear Schrödinger equations, as $Q$ is nilpotent or semisimple. In the KdV case, for example, a solution to

$$
4 u_{t}-u_{x x x}-6 u u_{x}=0
$$

gives a solution to the reduced equations such that $u=-\operatorname{tr}\left(P^{2}\right)$.

EXAMPLE 2. Here we have three symmetry generators in space-time, $X=\partial_{w}, Y=$ $\partial_{\tilde{z}}+\partial_{z}$, and $Z=(z-\tilde{z}) \partial_{w}+\tilde{w}\left(\partial_{\tilde{z}}-\partial_{z}\right)+\partial_{\tilde{w}}$, and the self-duality equations can be written in terms of the corresponding Higgs fields $P(t), Q(t), R(t)$ in the form

$$
Q^{\prime}=[R, P], \quad R^{\prime}=[t P+R, Q],
$$

where $t=\tilde{z}-z-\tilde{w}^{2}$. When the gauge group is $\operatorname{SL}(2, \mathbb{C})$ and $P$ is nilpotent, we have that the roots of $\operatorname{det}([P, y Q-R])=0$ satisfy the first Painlevé equation (Ince 1958, Mason and Woodhouse 1993)

$$
y^{\prime \prime}=6 y^{2}+t .
$$

When $\operatorname{det} P \neq 0$, the reduction is the second Painlevé equation.

3. Hierarchies of flows. Many of the characteristic features of integrable systems emerge in the reduction process from the geometry of the self-duality equations. For example, infinite hierarchies of commuting flows can be obtained from a natural recursion operator on the solutions to the linearized self-duality equation. If we choose a gauge in which the $w$ and $z$ components of $\Phi$ vanish, then we can write $\Phi$ in either of the forms

$$
\Phi=J^{-1} J_{\tilde{w}} \mathrm{~d} \tilde{w}+J^{-1} J_{\tilde{z}} \mathrm{~d} \tilde{z}=K_{z} \mathrm{~d} \tilde{w}+K_{w} \mathrm{~d} \tilde{z},
$$

where $J$ and $K$ are matrix-valued functions of the space-time coordinates and the subscripts denote partial derivatives. This leads to two equivalent potential forms of the SDYM equation:

$$
\left(J^{-1} J_{\tilde{w}}\right)_{w}-\left(J^{-1} J_{\tilde{z}}\right)_{z}=0 \quad \text { or } \quad K_{z \tilde{z}}-K_{w \tilde{w}}+\left[K_{w}, K_{z}\right]=0
$$

(Yang 1977, Newman 1978). The key to the construction is that the linearized forms of both of these are equivalent to the background-coupled wave equation $\mathrm{D} * \mathrm{D} \phi=0$, where $\phi=\delta K$ or $\phi=J^{-1} \delta J$. From $\Phi$ and a given solution $\delta \Phi$ to the linearized self-duality equation, one can construct $J$ and $\delta J$. Then by putting $\delta K=J^{-1} \delta J$, one obtains a new linearized solution. The recursion operator $R$ is the linear mapping in the tangent space to the manifold $\mathcal{M}$ of solutions to the SDYM equations (connections modulo gauge) determined by this construction (in some contexts, it is important to keep in mind that the definition of $R$ involves the choice of constants of integration). If one starts with the 'seed flows' on $\mathcal{M}$ given by the translations in space-time, and repeatedly applies $R$ at each solution to the tangent vector field to the flow on $\mathcal{M}$, then the result is a hierarchy of vector fields that generate new flows on $\mathcal{M}$. It is a direct consequence of the twistor 
construction that these commute, and it can be shown in examples that they reduce to the known hierarchies associated with standard integrable systems.

4. Bäcklund transformations. One can also generate many of the discrete symmetries of integrable equations from Bäcklund transformations of the $J$-form of the selfduality equation. If $J$ is a generic $n \times n$ matrix, then we can split it into blocks by writing $n=k+\tilde{k}$ and decomposing it as the product

$$
J=\left(\begin{array}{cc}
1 & \tilde{B} \\
0 & \tilde{A}^{-1}
\end{array}\right)^{-1}\left(\begin{array}{cc}
A^{-1} & 0 \\
B & 1
\end{array}\right),
$$

where $A$ is a $k \times k$ matrix, $\tilde{A}$ is a $\tilde{k} \times \tilde{k}$ matrix, and so on. One then determines a new solution $J^{\prime}$ from the given one $J$ by putting $A^{\prime}=\tilde{A}^{-1}, \tilde{A}^{\prime}=A^{-1}$ and by solving the linear equations

$$
\begin{array}{ll}
\tilde{B}_{\tilde{z}}^{\prime}=\tilde{A} B_{w} A, & \tilde{B}_{\tilde{w}}^{\prime}=\tilde{A} B_{z} A \\
B_{z}^{\prime}=A \tilde{B}_{\tilde{w}} \tilde{A}, & B_{w}^{\prime}=A \tilde{B}_{\tilde{z}} \tilde{A}
\end{array}
$$

for the new matrices $B^{\prime}$ and $\tilde{B}^{\prime}$. These are integrable as a consequence of the SDYM equations, and the new matrix $J^{\prime}$ constructed from $A^{\prime}, \tilde{A}^{\prime}, B^{\prime}$, and $\tilde{B}^{\prime}$ is again a solution (Mason et al. 1988, Woodhouse and Mason 1988).

5. The twistor construction. The twistor construction solves the SDYM equation in terms of a free holomorphic matrix-valued function of three variables, $P(\lambda, \mu, \zeta)$, from which $J$ is recovered by solving a Riemann-Hilbert problem (Ward 1977). One makes the substitution $\lambda=w \zeta+\tilde{z}, \mu=z \zeta+\tilde{w}$, and regards $P$ as a function of $\zeta$ for fixed values of the space-time coordinates. Then, provided that $P$ is nonsingular in a neighbourhood of the unit circle in the $\zeta$-plane, one can look for a splitting of the form $P=\tilde{H}^{-1}(\zeta) H(\zeta)$, where $H$ is holomorphic with respect to $\zeta$ and has nonvanishing determinant inside the unit disc and $\tilde{H}$ is holomorphic and has nonvanishing determinant outside the unit disc, including the point at infinity. If such a splitting exists at one space-time point, then it exists in a neighbourhood of that point and $J=\tilde{H}^{-1}(\infty) H(0)$ satisfies the $J$-form of the self-duality equation as a function of the space-time coordinates.

Much of the theory of integrable systems takes a particularly simple form when it is expressed in terms of this 'patching matrix' $P$ (so called because it has a geometric interpretation as the patching matrix of a holomorphic vector bundle over part of $\mathbb{C P}_{3}$ ). For example, the Bäcklund transformation becomes the algebraic operation

$$
P \mapsto\left(\begin{array}{cc}
0 & -1_{k} \\
\zeta^{-1} 1_{\tilde{k}} & 0
\end{array}\right)^{-1} P\left(\begin{array}{cc}
0 & -1_{k} \\
\zeta^{-1} 1_{\tilde{k}} & 0
\end{array}\right)
$$

where $1_{k}$ and $1_{\tilde{k}}$ are the $k \times k$ and $\tilde{k} \times \tilde{k}$ identity matrices; the recursion operator becomes $\delta P \mapsto \zeta \delta P$; and the flows generated from translation by recursion become

$$
P(\lambda, \mu, \zeta) \mapsto P(\lambda+\alpha, \mu+\beta, \zeta),
$$


where $\alpha$ and $\beta$ are holomorphic functions of $\zeta$ alone on some annular neighbourhood of the unit circle. The coefficients in the Laurent expansions of $\alpha$ and $\beta$ are the 'time' parameters along the flows.

6. The SDYM equations and Einstein's equations. Two apparently very distinct connections have been thoroughly explored and are now well understood. The first is between the reduced vacuum equation for real space-times with two commuting Killing vectors and a similar reduction of the SDYM equation in Minkowski space. The second is between the vacuum equation for (necessarily complex or non-Lorentzian) metrics with self-dual conformal structure and the self-duality equations with infinite-dimensional gauge groups. Both make it possible to apply twistor methods to the vacuum equations. The second connection, while at first sight of less direct physical relevance, is deeper and suggestive of generalizations of twistor methods that might encompass the full equations without requiring symmetry or self-duality assumptions. We shall look at each in turn.

7. Vacuum space-times with two commuting Killing vectors. If $X_{(i)}^{a}, i=1,2$, are commuting Killing vectors generating an orthogonally transitive action on a spacetime with metric $g_{a b}$, then

$$
R_{a b} X_{(i)}^{a} X_{(j)}^{b}=-\frac{1}{2} J_{i k} g^{-1 / 2} \partial_{a}\left(g^{1 / 2} g^{a b} J^{k l} \partial_{b} J_{j l}\right),
$$

where $J=\left(J_{i j}\right)=\left(X_{(i)}^{a} X_{(j) a}\right)$ and $\left(J^{i j}\right)$ is the inverse matrix. So if $g_{a b}$ is a solution to the vacuum equation, then

$$
D_{a}\left(r J^{-1} D^{a} J\right)=0
$$

where $D$ is the intrinsic covariant derivative on a 2 -surface $\Sigma$ orthogonal to the orbits, and $r^{2}=-\operatorname{det} J$ (we assume that $r$ is not constant and that the orbits are not null). Conversely, eqn (1) is conformally invariant on $\Sigma$, and any solution, with $J$ symmetric and satisfying the constraint on its determinant, determines a solution to the vacuum equations: the remaining metric components are found by direct integration.

The same equation arises by identifying $\Sigma$ (as a 2-dimensional conformal manifold) with the quotient of Minkowski space by a two-dimensional isometry group and reducing the $J$-form of the SDYM equations, in either of the two cases in which the metric is written in the respective forms

$$
\mathrm{d} t^{2}-\mathrm{d} z^{2}-\mathrm{d} r^{2}-r^{2} \mathrm{~d} \theta^{2} \quad \text { or } \quad \mathrm{d} u^{2}-u^{2} \mathrm{~d} \chi^{2}-\mathrm{d} v^{2}-v^{2} \mathrm{~d} \theta^{2},
$$

and the groups are generated by $\partial_{t}, \partial_{\theta}$ in the first case and by $\partial_{\theta}, \partial_{\chi}$ in the second (Witten 1979, Ward 1983, Fletcher and Woodhouse 1990). Thus reductions of the selfdual Yang-Mills equations (subject to certain constraints that ensure that $J$ is real, symmetric, and has the correct determinant) by either of these two groups give rise to solutions of Einstein's equations. Similar statements hold in the case of the EinsteinMaxwell equations, except that $J$ is then a $3 \times 3$ matrix constructed from the metric and the electromagnetic potential (Woodhouse 1990a). 
8. The gauge group of volume preserving transformations. The SDYM equation on a connection invariant under the translation group in complex Minkowski space takes the form $[L, M]=0$, where

$$
L=W-\zeta \tilde{Z}, \quad M=Z-\zeta \tilde{W}
$$

and $W, Z, \tilde{W}$, and $\tilde{Z}$ are the Higgs fields of the translations in the four coordinate directions: they are constant elements of the Lie algebra of the gauge group. The self-dual vacuum equation on a complex metric can be put in this form by taking as gauge group the group $G$ of diffeomorphisms of a four-manifold $M$ that preserve a given volume element $\Omega$. Then the Higgs fields are divergence-free vector fields on $M$, and the self-duality equation is precisely the condition that $\alpha^{-1} W, \alpha^{-1} Z, \alpha^{-1} \tilde{W}$, and $\alpha^{-1} \tilde{Z}$ should be a null tetrad for a self-dual vacuum metric, where $\alpha^{2}=\Omega(W, Z, \tilde{W}, \tilde{Z})$. Every such metric arises in this way (Mason and Newman 1989, Chakravarty et al. 1991, see also Ashtekar et al. 1988 and Ward 1990b, 1992). ${ }^{1}$

9. What can you do with this? This chain of ideas opens the door to interesting applications in relativity of twistor methods and of techniques from the theory of integrable systems. They come under three headings:

Solution generation: by passing from well-known solutions to the standard integrable equations (KdV, Painlevé, ... ) to Yang-Mills fields, one can construct new solutions to the self-dual vacuum equation.

Hidden symmetries: the twistor representation can make obvious symmetries of the Einstein equations that are hard to see directly. For example, in the case of vacuum solutions with two commuting Killing vectors, the group generated by $J \mapsto J^{\prime}$ (with $k=\tilde{k}=1$ ) and $J \mapsto A J A^{t}$ (with A constant), that is the 'Geroch group', is given by a natural action of a loop group on the corresponding bundles over twistor space (Woodhouse and Mason 1988, Kinnersley 1971, Geroch 1971, 1972, Kinnersley and Chitre 1977-8; see also Hoensalears and Dietz 1984).

Global geometry: a characteristic of twistor constructions is that they 'solve' nonlinear equations in terms of free holomorphic data. In all but the simplest cases, it is not possible to extract the solution explicitly, but it may nonetheless be possible to read off some local and global properties of the solution from the data.

More intriguingly, it allows glimpses of the way in which twistor techniques might be extended and adapted to encompass not just these two special cases of Einstein's equations, but also the full equations without any special simplifying assumptions: at both ends of the chain, we have the four-dimensional geometry of space-time (the flat

\footnotetext{
${ }^{1}$ The original application of twistor methods to the self-dual vacuum equation was by Penrose (1976). His construction was reinterpreted in a Riemannian context by Atiyah et al (1978).
} 
space-time on which the Yang-Mills field is defined, and the curved space-time of the solution to Einstein's equations).

We shall now look at some examples, and, briefly, at some ideas on how the techniques might be extended to the general case.

10. KdV solutions to the self-dual vacuum equations. A solution to the $\mathrm{KdV}$ equation determines a self-dual connection $\mathrm{D}$ on a rank-2 vector bundle $E \rightarrow U$ over an open set $U$ in complex space-time, invariant under translation along $X=\partial_{w}-\partial_{\tilde{w}}$ and $Y=\partial_{\tilde{z}}$. That is, $X$ and $Y$ lift to vector fields $X^{\prime}$ and $Y^{\prime}$ on $E$, the flow of which preserves the horizontal subspaces of D. From these objects, we construct a self-dual vacuum metric on the four-dimensional quotient space $M$ of $E$ by the flows along $X^{\prime}$ and $Y^{\prime}$. First we construct a volume element $\Omega$ on $M$ by pushing down the contraction of the natural 6-form on $E$ with $X^{\prime}$ and $Y^{\prime}$ (the 6-form is the product of the Minkowski volume element on $U$ and the $\mathrm{SL}(2, \mathbb{C})$-invariant 2-form on the fibres of $E)$. We then construct the metric on $M$ by lifting the four coordinate vectors $\partial_{w}, \partial_{z}, \partial_{\tilde{w}}$ and $\partial_{\tilde{z}}$ in flat space-time horizontally to $E$, where they commute with $X^{\prime}$ and $Y^{\prime}$, and then projecting them into $M$. The projected vector fields commute, and are divergence-free, and, because D is self-dual, their Lie brackets satisfy the correct commutation relations for a self-dual vacuum metric. It is not hard to write down the metric explicitly in terms of the original solution to the KdV equation, and to develop other examples by using different soliton equations (Mason and Woodhouse 1996, Dunajski et al. 1996; similar constructions appear in Maszczyk et al. 1994 and in Maszczyk 1995).

11. Painlevé solutions to the Ernst equation. If we write the flat space-time metric in the form

$$
\mathrm{d} s^{2}=\mathrm{d} u^{2}-u^{2} \mathrm{~d} \chi^{2}-\mathrm{d} v^{2}-v^{2} \mathrm{~d} \theta^{2},
$$

then solutions to the reduced vacuum equations for metrics with two orthogonally commuting Killing vectors correspond to solutions of the $\mathrm{SL}(2, \mathbb{C}) \mathrm{SDYM}$ equations that are invariant under the flat-space isometries generated by $\partial_{\theta}$ and $\partial_{\chi}$ (and have certain other discrete symmetries); on the other hand, the reduction of the self-dual Yang-Mills equation by the three conformal isometries generated by $\partial_{\theta}, \partial_{\chi}$, and $u \partial_{u}+v \partial_{v}$ is the sixth Painlevé equation. By following through this correspondence, one can construct vacuum solutions from the corresponding Painlevé transcendents (Calvert and Woodhouse 1996). One can do the same thing with solutions to the third Painlevé equation, which, as in the example above, correspond to solutions of the self-dual Yang-Mills equations invariant under flows of $\partial_{t}, \partial_{z}$, and $\partial_{\theta}$, where now the flat-space metric is written in the cylindrical-polar form

$$
\mathrm{d} s^{2}=\mathrm{d} t^{2}-\mathrm{d} z^{2}-\mathrm{d} r^{2}-r^{2} \mathrm{~d} \theta^{2} .
$$

In this case, one exploits the fact that the reduced Einstein equations are equivalent to the stationary axisymmetric reduction of the SDYM equation. 
The existence of such solutions, which is a natural signature of the integrability of the Ernst equation, was observed, using other means, by Cosgrove (1977) in the case of $\mathrm{P}_{\mathrm{VI}}$, and by Persides and Xanthopoulos (1988), Chandrasekhar (1986), and Léauté and Marcilhacy (1979) in the case of $\mathrm{P}_{\mathrm{III}}$ (which is the same as a special case of $\mathrm{P}_{\mathrm{V}}$ ); see also Ablowitz and Clarkson (1991). One can also construct self-dual conformal structures and Einstein metrics from Painlevé transcendents (Hitchin 1995, Tod 1994, Maszczyk et al. 1994).

12. Global properties of stationary axisymmetric solutions. By combining the twistor construction with the identification of the reduced Einstein equations with the reduced SDYM equations, one can generate stationary axisymmetric vacuum metrics from holomorphic functions $P(\gamma)$ of a single complex variable: $P$ must take values in $\mathrm{SL}(2, \mathbb{C})$, and must be symmetric and real in the sense that $\overline{P(\gamma)}=P(\bar{\gamma})$ (geometrically, $P$ is the transition matrix of a holomorphic vector bundle over a one-dimensional reduced twistor space). The solution is recovered by substituting

$$
\gamma=\frac{1}{2} r\left(\zeta^{-1}-\zeta\right)+z
$$

fixing $r, z$, and solving the Riemann-Hilbert problem $P(\zeta)=\tilde{H}(\zeta)^{-1} H(\zeta)$, in the same way as before, and by writing

$$
J^{\prime}=\tilde{H}^{-1}(\infty) H(0)=\frac{1}{f}\left(\begin{array}{cc}
1 & -\psi \\
-\psi & f^{2}+\psi^{2}
\end{array}\right)
$$

(this matrix $J^{\prime}$ is related to the matrix of inner products of the Killing vectors by a Bäcklund transformation). Then $\mathcal{E}=f+\mathrm{i} \psi$ is a solution to the Ernst equation. Although in principle all solutions can be obtained in this way, in practice it is very hard to solve the Riemann-Hilbert problem explicitly. The important points, however, are that the solution can be described implicitly by free data, and that many global properties of the solution can be seen without actually finding an analytic expression for the metric. For example, asymptotic flatness (without 'NUT-like behaviour') implies that $P$ should be holomorphic at $\gamma=\infty$, with its off-diagonal entries falling off like $\gamma^{-2}$; and the poles in $P$ can be identified with axis-horizon intersection points. From these, one can see that the only possible black hole solution (asymptotically flat with two such intersection points) is generated by

$$
P=\frac{1}{\gamma^{2}-\sigma^{2}}\left(\begin{array}{cc}
(\gamma+m)^{2}+a^{2} & 2 a m \\
2 a m & (\gamma-m)^{2}+a^{2}
\end{array}\right),
$$

where $\sigma^{2}=m^{2}-a^{2}$. This is the generator of the Kerr metric (Fletcher 1990, Fletcher and Woodhouse 1990).

13. Propagation from initial data. In the case of cylindrical symmetry, one can derive the matrix $P(\gamma)$ from the initial data. In this case, the reduced vacuum equations 
come down to the nonlinear wave equation

$$
\left(r J^{\prime-1} J_{t}^{\prime}\right)_{t}-\left(r J^{\prime-1} J_{r}^{\prime}\right)_{r}=0
$$

on the the $2 \times 2$ matrix $J^{\prime}$ in (2), where $r$ and $t$ are the nonignorable coordinates. One can generate solutions in the same way as in the stationary axisymmetric case, except that now one substitutes $\gamma=t+r \sin \theta$, where $\zeta=\mathrm{e}^{\mathrm{i} \theta}$, before solving the Riemann-Hilbert problem. This apparently slight difference has two important consequences. The first is that it is only necessary to know $P$ for real values of $\gamma$ in order to obtain a solution: $P$ and the resulting metric need not be analytic (Woodhouse 1989). The second point (which I did not realise until more recently) concerns the global behaviour of the solution. For fixed $r, t$, we have that $P(r+t \sin \theta)$ is a symmetric matrix-valued function on the unit circle in the complex plane. However, it is not always possible to find holomorphic matrices $\tilde{H}$ and $H$ on the outside and inside of the unit circle such that $P=\tilde{H}^{-1} H$. If it is possible at one point $(r, t)$, then it is possible at nearby points; but in general, one expects 'jumping points', where the splitting fails, and the corresponding metric has algebraic singularities. However this cannot happen if $P$ is positive definite for real $\gamma$, as a consequence of an old theorem of Gohberg and Krein (1958); a proof is given in Mason and Woodhouse (1996): if $P$ is real and positive definite on the real axis in the $\gamma$-plane, then $J^{\prime}(r, t)$ is nonsingular for all $r$ and $t$.

One can find $P$ from the Cauchy data $J^{\prime}(r, 0), J_{t}^{\prime}(r, 0)$ by solving an ordinary differential equation. We take $A(r)$ and $B(r)$ to be, respectively, the odd and even functions of $r$ such that $A=J^{\prime-1} J_{r}^{\prime}$ and $B=J^{\prime-1} J_{t}^{\prime}$ for $t=0, r \geq 0$. We then solve, for the matrix $S(\gamma, \theta)$, the equation

$$
\partial_{\theta} S=\frac{1}{2} S((1+\cos \theta) A(\gamma \sin \theta)+B(\gamma \sin \theta) \sin \theta), \quad S(\gamma, 0)=1 .
$$

Then the solution is generated throughout the $r, t$ plane by

$$
P(\gamma)=J^{\prime}(0,0) S(\gamma, \pi / 2) J^{\prime}(\gamma, 0)^{-1} S(\gamma, \pi / 2)^{\mathrm{t}} J^{\prime}(0,0)^{\mathrm{t}} .
$$

If the Cauchy data are such that $P$ is smooth and $J^{\prime}(\gamma, 0)$ is nonsingular, then the solution is regular for all $r, t$. This gives an alternative starting point for establishing the long-time existence theorems that underlie the results on the global propagation of solutions with cylindrical symmetry in Berger et al. (1995).

L. J. Mason has given, in a similar spirit, a method for finding by twistor methods the metric in the interaction region of two colliding plane waves from data on the wave fronts (see Woodhouse 1990b).

14. The spin $3 / 2$ equation. These methods apply so far only to metrics with a high degree of symmetry (about which much can and has been been discovered by other older means) and to metrics with self-dual conformal structures, which do not have a direct physical interpretation in classical general relativity (but which are nonetheless useful for exploring properties of the field equations). We are optimistic that it will be possible to 
extend them. The most promising line of attack is through exploiting the consistency of the potential form of the spin-3/2 massless wave equation in a curved background as a 'linear system' for the vacuum equation $R_{a b}=0$.

In a space-time with spin structure, there are two natural $\mathrm{SL}(2, \mathbb{C})$-vector bundles $S, S^{\prime} \rightarrow M$ and a natural map $\Gamma\left(S^{\prime}\right) \rightarrow \Omega^{1}(S)$. (In two-component notation, $S$ and $S^{\prime}$ are the bundles of unprimed and primed spinors and the map from sections of $S^{\prime}$ to 1 -forms with values in $S$ is given by $\alpha^{A^{\prime}} \mapsto \alpha_{A^{\prime}} \mathrm{d} x^{A A^{\prime}}$ ). In terms of these objects, the vacuum equation is equivalent to the vanishing of the composite of the maps:

$$
\Gamma\left(S^{\prime}\right) \stackrel{D}{\longrightarrow} \Omega^{1}\left(S^{\prime}\right) \stackrel{D}{\longrightarrow} \Omega^{2}\left(S^{\prime}\right) \longrightarrow \Omega^{3}(S),
$$

where $D$ is the covariant exterior derivative. In the flat case $\sigma \in \Omega^{1}\left(S^{\prime}\right) \mapsto 0 \in \Omega^{3}(S)$ is the Rarita-Schwinger equation, $D \sigma$ is a spin-3/2 field, and the twistor space has a natural interpretation as a space of charges for such fields (Penrose 1992). L. J. Mason and J.-P. Nicholas have recently begun work on using this formulation of the field equation, in a $3+1$ decomposition, as a tool for investigating the long-time propagation of the full vacuum equations, in a way that might be seen as extending the results on the propagation of cylindrical waves.

\section{References}

M. J. Ablowitz and P. A. Clarkson (1991), Solitons, nonlinear evolution equations and inverse scattering. London Mathematical Society Lecture Notes in Mathematics, 149, Cambridge University Press, Cambridge.

A. Ashtekar, T. Jacobson and L. Smolin (1988), A new characterization of half-flat solutions to Einstein's equation. Commun. Math. Phys., 115, 631-48.

M. F. Atiyah, N. J. Hitchin and I. M. Singer (1978), Self-duality in four-dimensional Riemannian geometry. Proc. Roy. Soc. Lond., A 362, 425-61.

M. F. Atiyah and R. S. Ward (1977), Instantons and algebraic geometry. Commun. Math. Phys., 55, 111-24.

B. K. Berger, P. T. Chruściel and V. Moncrief (1995), On "asymptotically flat" spacetimes with $G_{2}$-invariant Cauchy surfaces. Ann. Phys., 237, 322-54.

G. Calvert and N. M. J. Woodhouse (1996), Painlevé transcendents and Einstein's equation. Class. Quantum Grav., 13, L33-9.

S. Chakravarty, L. Mason J. and E. T. Newman (1991), Canonical structures on anti-selfdual four-manifolds and the diffeomorphism group. J. Math. Phys., 32, 1458-64.

S. Chandrasekhar (1986), Cylindrical gravitational waves. Proc. Roy. Soc. Lond., A408, 20932.

C. M. Cosgrove (1977), New family of exact stationary axisymmetric gravitational fields generalising the Tomimatsu-Sato solutions. J. Phys., A10, 1481-524.

M. Dunajski, L. J. Mason and N. M. J. Woodhouse (1996), From 2D integrable systems to self-dual gravity. In preparation. 
J. Fletcher (1990), Non-Hausdorff twistor spaces and the global geometry of space-time. D. Phil. thesis, University of Oxford.

J. Fletcher and N. M. J. Woodhouse (1990), Twistor characterization of stationary axisymmetric solutions of Einstein's equations. In Twistors in mathematics and physics. Eds. T. N. Bailey and R. J. Baston. London Mathematical Society Lecture Notes in Mathematics, 156. Cambridge University Press, Cambridge.

R. Geroch (1971), A method for generating new solutions of Einstein's equations. J. Math. Phys., 12, 918-24.

R. Geroch (1972), A method for generating new solutions of Einstein's equations, II. J. Math. Phys., 13, 394-404.

I. C. Gohberg and M. G. Krein (1958), Systems of integral equations on the half line with kernels depending on the difference of the arguments. Uspekhi Mat. Nauk, 13, 3-72. (Russian)

N. J. Hitchin (1995), Twistor spaces, Einstein metrics and isomonodromic deformations. J. Diff. Geom., 42, 30-112.

C. Hoenselaers and W. Dietz (eds.) (1984), Solutions of Einstein's equations: techniques and results. Lecture Notes in Physics, 205. Springer, Berlin.

E. L. Ince (1956), Ordinary differential equations. Dover, New York.

W. Kinnersley (1977), Symmetries of the stationary Einstein-Maxwell field equations, I. J. Math. Phys., 18, 1529-37.

W. Kinnersley and D. M. Chitre (1977-8), Symmetries of the stationary Einstein-Maxwell field equations, II-IV. J. Math. Phys., 18, 1538-42, 19, 1926-31, 2037-42.

B. Léauté and G. Marcilhacy (1979), Sur certaines particulières transcendantes des équations d'Einstein. Ann. Inst. H. Poincaré, 31, 363-75.

L. J. Mason, S. Chakravarty and E. T. Newman (1988), Bäcklund transformations for the anti-self-dual Yang-Mills equations. J. Math. Phys., 29, 4, 1005-13.

L. J. Mason and E. T. Newman (1989). A connection between the Einstein and Yang-Mills equations. Commun. Math. Phys., 121, 659-68.

L. J. Mason and G. A. J. Sparling (1989), Nonlinear Schrödinger and Korteweg de Vries are reductions of self-dual Yang-Mills. Phys. Lett., A137, 29-33.

L. J. Mason and N. M. J. Woodhouse (1993), Self-duality and the Painlevé transcendents. Nonlinearity, 6, 569-81.

L. J. Mason and N. M. J. Woodhouse (1996), Integrability, self-duality, and twistor theory. London Mathematical Society Monographs. Oxford University Press, Oxford.

R. Maszczyk (1995), The symmetry transformation - self-dual Yang-Mills fields and self-dual metrics. Ph. D. Thesis, Warsaw University.

R. Maszczyk, L. J. Mason and N. M. J. Woodhouse (1994), Self-dual Bianchi metrics and the Painlevé transcendents. Class. Quantum Grav., 11, 65-71.

E. T. Newman (1978), Source-free Yang-Mills theories. Phys. Rev., D18, 2901-2908.

R. Penrose (1976), Nonlinear gravitons and curved twistor theory. Gen. Rel. Grav., 7, 31-52.

R. Penrose (1992), Twistors as spin $3 / 2$ charges. In Gravitation and modern cosmology. Eds. A. Zichichi and N. Sánchez. Plenum Press, New York.

S. Persides and B. C. Xanthopoulos (1988), Some new stationary axisymmetric asymptotically flat space-times obtained from Painlevé transcendents. J. Math. Phys., 29, 674-80. 
K. P. Tod (1994), Self-dual Einstein metrics from the Painlevé VI equation. Phys. Lett., A190, $221-4$.

R. S. Ward (1977), On self-dual gauge fields. Phys. Lett., 61A, 81-2.

R. S. W ard (1983), Stationary axisymmetric space-times: a new approach. Gen. Rel. Grav., 15, 105-9.

R. S. Ward (1985), Integrable and solvable systems and relations among them. Phil. Trans. R. Soc., A315, 451-7.

R. S. Ward (1990a), Integrable systems in twistor theory. In Twistors in mathematics and physics. Eds. T. N. Bailey and R. J. Baston. London Mathematical Society Lecture Notes in Mathematics, 156. Cambridge University Press, Cambridge.

R. S. Ward (1990b), The SU( $\infty)$ chiral model and self-dual vacuum spaces. Class. Quantum Grav., 7, L217-22.

R. S. W ard (1992), Infinite-dimensional gauge groups and special nonlinear gravitons. J. Geom. Phys., 8, 317-25.

L. Witten (1979), Static axially symmetric solutions of self-dual SU(2) gauge fields in Euclidean four-dimensional space. Phys. Rev., D19, 718-20.

N. M. J. Woodhouse (1989), Cylindrical gravitational waves. Class. Quantum Grav., 6, 933-43.

N. M. J. Woodhouse (1990a), Ward's splitting construction for stationary axisymmetric solutions of the Einstein-Maxwell equations. Class. Quantum Grav., 7, 257-60.

N. M. J. Woodhouse (1990b), Spinors, twistors, and complex methods. In: General relativity and Gravitation 1989, pp. 93-8 (eds Ashby, Bartlett, and Wyss), CUP.

N. M. J. Woodhouse and L. J. Mason (1988), The Geroch group and non-Hausdorff twistor spaces. Nonlinearity, 1, 73-114.

C. N. Yang (1977), Condition of self-duality for SU(2) gauge fields on Euclidean four-dimensional space. Phys. Rev. Lett., 38, 1377-9. 